\title{
Comparison of residual strains measured by X-ray and neutron diffraction in a titanium $(\mathrm{Ti}-6 \mathrm{Al}-4 \mathrm{~V})$ matrix composite
}

\author{
Partha Rangaswamy a,*, Michael B. Prime a , Mark Daymond ${ }^{\mathrm{b}}$, M.A.M. Bourke ${ }^{\mathrm{a}}$, \\ Bjørn Clausen a, Hahn Choo $^{a}$, N. Jayaraman ${ }^{\mathrm{c}}$ \\ ${ }^{a}$ Los Alamos National Laboratory, Materials Science and Engineering, Lujan Center, H805, Los Alamos, Mexico \\ ${ }^{\mathrm{b}}$ ISIS, Rutherford Appleton Laboratory, Chilton, Didcot, Oxon, OX11 OQX, UK \\ ${ }^{\mathrm{c}}$ Department of Materials Science and Engineering, University of Cincinnati, Cincinnati, Ohio 45221-0012, USA
}

\begin{abstract}
This research compares matrix thermal residual strains measured in a continuous fiber reinforced $\mathrm{SiC} / \mathrm{Ti}-6 \mathrm{Al}-4 \mathrm{~V}$ titanium matrix composite (TMC) using X-ray and neutron diffraction with finite element predictions. The strain dependence on the strains for several reflections $(105,204,300,213$ and 312) of the matrix were explored at the surface (X-ray) and in the bulk (neutron). To determine the longitudinal surface strains from the X-ray measurements for comparison with the neutron values, the $\varepsilon_{\phi \psi}$ versus $\sin ^{2} \psi$ plots were extrapolated to $\psi=90^{\circ}$. Continuum micro-mechanics based multi-ply finite element models (FEM) simulating rectangular and hexagonal fiber distributions were explored for calculating average surface and bulk strains. For different reflections, the experimentally determined surface measured strains ranged from $+1904 \pm 424$ to $+2974 \pm 321 \mu \varepsilon$ and the bulk measurements ranged from $+2269 \pm 421$ to $+3022 \pm 1134 \mu \varepsilon$. These values contrast with the single valued FEM prediction of $+3200 \mu \varepsilon$ which was the same for both the surface and the bulk. (C) 1999 Elsevier Science S.A. All rights reserved.
\end{abstract}

Keywords: Residual stress; Neutron diffraction; Titanium matrix composites; Finite element analysis; X-ray measurement

\section{Introduction}

Residual strains in continuous fiber reinforced titanium alloy metal matrix composites (TMCs) are induced during the cooling from the consolidation temperature of $900^{\circ} \mathrm{C}$ and are caused by the 2-fold mismatch in the coefficient of thermal expansion (CTE) between the $\mathrm{SiC}$ fiber reinforcement $\left(3.2 \times 10^{-6} /{ }^{\circ} \mathrm{C}\right)$ and the titanium matrix alloy $\left(8.78 \times 10^{-6} /{ }^{\circ} \mathrm{C}\right)$. After cooling, the strain parallel to the fibers (longitudinal) is tensile in the matrix and compressive in the fiber. The magnitude, distribution and stability in service of the resulting residual strains and stresses influence the mechanical and physical properties of composites [1-4]. Despite extensive characterization [1-4] on TMC mechanical behavior, measurement and modeling of residual strains and stresses is a growing challenge. Models that predict residual stresses and their effect on lifetime predictions have to be validated $[4,5]$. Therefore, it is

\footnotetext{
* Corresponding author. E-mail: partha@lanl.gov (http:// www.lansce.lanl.gov/mlnsc)
}

important for residual strains and stresses to be experimentally determined and accounted for to achieve reliable design parameters.

This research explores the range of strains that are measurable depending on the selection of a particular lattice reflection and whether surface or bulk penetrating techniques are used.

\subsection{Review of diffraction measurements of strain in $\mathrm{SiC} / \mathrm{Ti} \mathrm{TMCS}$}

X-ray (XRD) [6-10] and neutron diffraction [10-16] are commonly used to determine residual stresses in continuous fiber reinforced TMCs. XRD is routinely used to monitor residual stresses in the matrix of TMCs, but because of the small penetration depth exhibited by soft X-rays [17], is limited to the near surface region $(<50 \mu \mathrm{m})$. By contrast, neutron diffraction due to its deep penetration can determine the strains in both the matrix and fiber simultaneously [16]. In this paper we focus only on the matrix strains. 


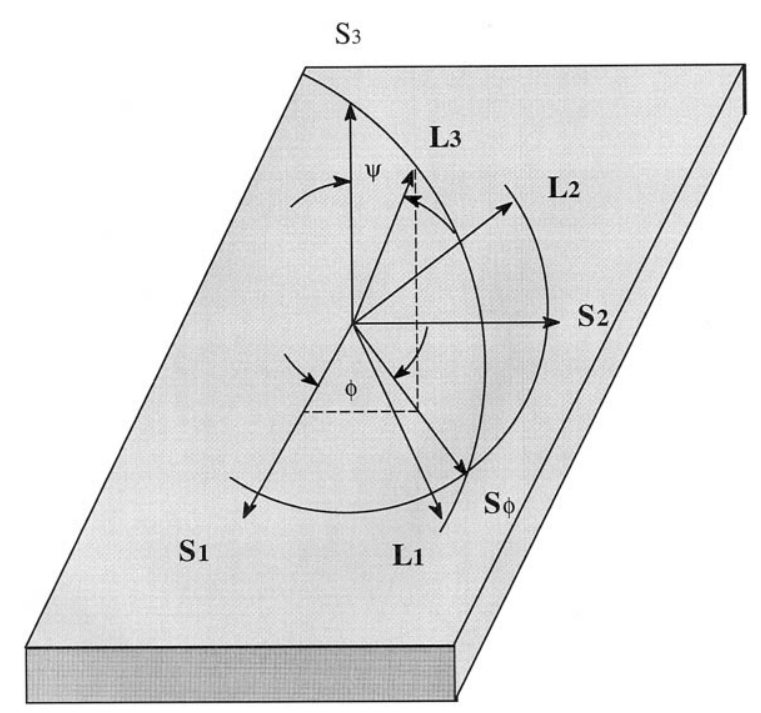

Fig. 1. Coordinate system used in the X-ray diffraction strain analysis. $\mathrm{S}_{1}, \mathrm{~S}_{2}$, and $\mathrm{S}_{3}$ are directions in the sample coordinate system parallel, transverse and normal to the fiber orientation.

In the past, there has been considerable focus on comparisons of experimentally determined stresses with finite element predictions [1,6-16]. Where comparisons have been made, it is often convenient to assume that a single measured reflection, with a suitable plane specific elastic constant is representative of the stress (surface or bulk) of the composite constituents, so a direct com-
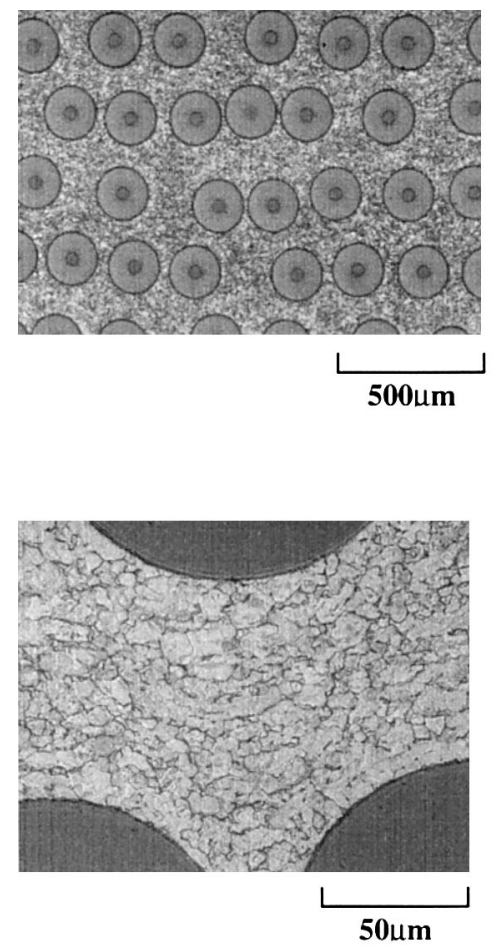

parison can be made with continuum mechanics based model predictions $[1,7,8,13,16]$. Although this approach is certainly valid for comparative measurements, care must be taken for comparisons of absolute magnitudes because of the range of elastic and plastic anisotropic effects present at the microstructure level.

In the X-ray technique, because of the assumptions of plane stress at the surface (generally satisfied for TMCs [6-8]), stresses are determined directly using the $\sin ^{2} \psi$ method, where the change in the interplanar atomic spacing is related to the stress through an X-ray elastic constant [17]. However, in a neutron measurement (steady state [12] or pulsed [11]), strains are calculated relative to a strain free standard. Since the calculation of stress in any direction requires strain measurements in at least three directions, this increases the time for the measurements and potentially results in compounding the errors.

Most XRD strain measurements use one reflection, the selection of which is typically arbitrary, provided that it is in the high angle $\left(\geq 120^{\circ} 2 \theta\right)$ region to minimize the systematic errors associated with beam optics and alignment with respect to the strain shifts [17]. In this study, strains for several hkls present in the high angle region are compared with similar hkls from neutron measurements. Since the two techniques rely on the same physics, the measurements should provide a comparison of strains at the surface and bulk and their sensitivity to choice of hkl [18].

(a)
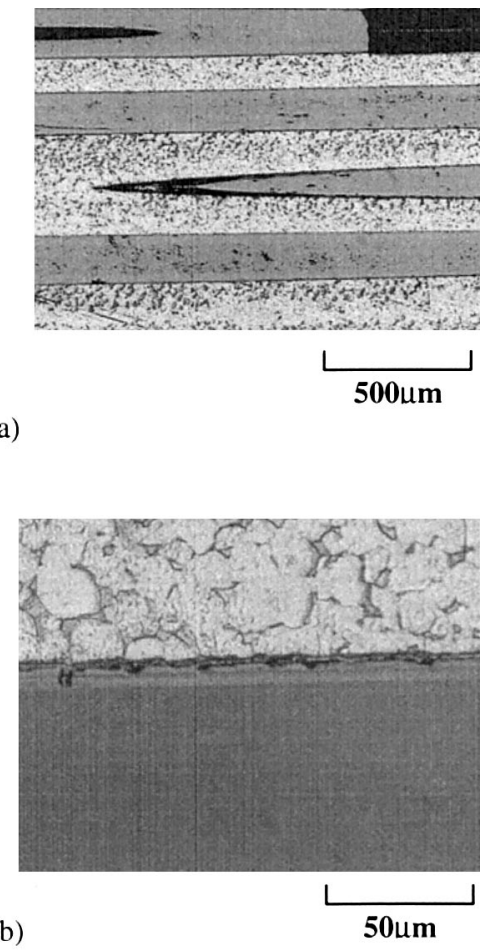

(b)

Fig. 2. Photomicrographs at perpendicular and parallel cross-sections to the fibers. (a) Typical fiber array spacing; $v_{\mathrm{f}} \approx 0.37$. Photograph at low magnification of $50 \times$. (b) Typical homogeneous, equiaxed $\alpha+\beta$ microstructure of the Ti-6Al-4V matrix alloy of the composite. Photograph at high magnification of $500 \times$. 


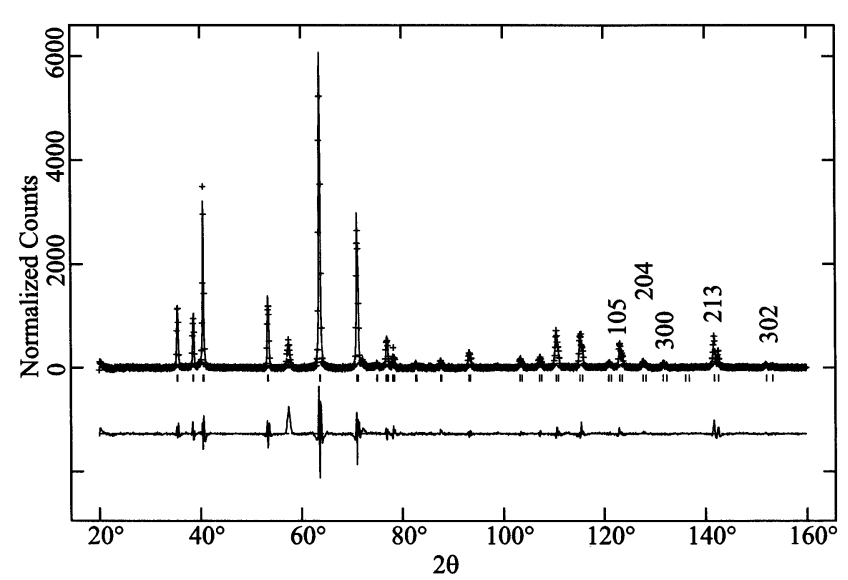

Fig. 3. X-ray diffraction spectra $\left(\mathrm{CuK}_{\alpha}\right)$ for the monolithic Ti-6Al$4 \mathrm{~V}$ showing the hkls 105, 204, 300, 213 and 302 used for strain measurements.

\subsection{Diffraction for residual strain measurements}

Diffraction based stress analysis techniques use distances between atomic planes of a crystalline specimen as an internal elastic strain gauge [17]. The fundamental relation for determining a general strain state with diffraction is [17]:

$$
\begin{aligned}
\varepsilon_{\phi \psi}= & \frac{d_{\phi \psi}-d_{0}}{d_{0}} \\
= & \varepsilon_{11} \cos ^{2} \phi \sin ^{2} \psi+\varepsilon_{12} \sin 2 \phi \sin ^{2} \psi+\varepsilon_{22} \sin ^{2} \phi \sin ^{2} \psi \\
& +\varepsilon_{33} \cos ^{2} \psi+\varepsilon_{13} \cos \phi \sin 2 \psi+\varepsilon_{23} \sin \phi \sin 2 \psi
\end{aligned}
$$

Here, $\varepsilon_{\phi \psi}$ is the strain in the direction given by the angles $\phi$ and $\psi$ and defined in Fig. 1. $\varepsilon_{\phi \psi}$ is determined from the interplanar spacing between the atomic planes $\left(d_{\phi \psi}\right)$ and the stress free spacing $\left(d_{0}\right)$. The strain components on the right hand side of Eq. (1) are referenced to the sample co-ordinate system, $S_{i}$, as shown in Fig. 1. The interplanar spacing $d_{\phi \psi}$ is determined using Bragg's law $\lambda=2 d \sin \theta$, where $\lambda$ is the wavelength, $d$ is the interplanar spacing, and $\theta$ is the diffracting angle. Usually, in the conventional $\mathrm{X}$ ray technique, $\lambda$ is fixed and $\theta$ is varied to determine the spacing of diffracting plane $\left(d_{\mathrm{hkl}}\right)$ (this also applies at a monochromatic neutron source). At a pulsed neutron source, $\theta$ is fixed and $\lambda$ is varied to determine $d_{\phi \psi}$. By measuring the strain $\varepsilon_{\phi \psi}$ in at least six independent directions, the six components of strain in the sample coordinate system can be determined, thus characterizing the strain tensor at the measurement location.

In a fiber reinforced TMC system, the principal strain axes are typically assumed to be parallel and perpendicular to the fiber directions. Since the princi- pal strain axes are assumed, the sample coordinate system $\left(S_{i}\right)$ shown in Fig. 1 can be matched with the directions of the principal axes. The shear strain components will be zero and the principal strains, $\varepsilon_{1}, \varepsilon_{2}$, $\varepsilon_{3}$ will correspond to the strains $\varepsilon_{11}$ (parallel to the fibers), $\varepsilon_{22}$ (in-plane perpendicular to the fibers), and $\varepsilon_{33}$ (out of plane perpendicular to the fibers), respectively, and can be determined by means of $\varepsilon_{\phi \psi}$ at $(\phi, \psi)$ equal to 0,$90 ; 90,90$; and 0,0 . The fundamental equation now reduces to:

$\varepsilon_{\phi \psi}=\frac{d_{\phi \psi}-d_{0}}{d_{0}}=\left(\varepsilon_{11} \cos ^{2} \phi+\varepsilon_{22} \sin ^{2} \phi-\varepsilon_{33}\right) \sin ^{2} \psi+\varepsilon_{33}$

Since we are interested in the strains parallel to the fibers $\left(\varepsilon_{11}\right)$ where $\phi=0^{\circ}$, Eq. (2) reduces to (Fig. 1):

$\varepsilon_{\phi \psi}=\frac{d_{\phi \psi}-d_{0}}{d_{0}}=\left(\varepsilon_{11}-\varepsilon_{33}\right) \sin ^{2} \psi+\varepsilon_{33}$

In practice, $\varepsilon_{\phi \psi}$ versus $\sin ^{2} \psi$ is plotted by obtaining $\varepsilon_{\phi \psi}$ at $\psi$ angles varying from 0 to $60^{\circ}$ to obtain the slope $\left(\varepsilon_{11}-\varepsilon_{33}\right)$ [17]. If the variation of $\varepsilon_{\phi \psi}$ versus $\sin ^{2} \psi$ plot is linear, then it is an indication of a plane stress condition at the surface, since the depth of penetration is also very small $\left(<12\right.$ microns at $\psi=0^{\circ}$ using $\mathrm{CuK}_{\alpha}$ ).

Using generalized Hooke's law for stress measurement, Eq. (3) is converted to stress $\sigma_{11}$ parallel to the fibers by:

$\sigma_{11}=\frac{E}{1+v}\left(\varepsilon_{11}-\varepsilon_{33}\right)$

Knowing the slope $\left(\varepsilon_{11}-\varepsilon_{33}\right)$ from Eq. (3) and the XRD elastic constant $(E / 1+v)$ for the hkl diffracting plane used, in-plane stress $\left(\sigma_{11}\right)$ is obtained by the conventional practice of calculating stresses from XRD technique [17].

However in this study we were interested in the in-plane surface strains. After verifying that the $\varepsilon_{\phi \psi}$ versus $\sin ^{2} \psi$ plot is linear, we obtain this by extrapolating the $\varepsilon_{\phi \psi}$ versus $\sin ^{2} \psi$ plot to $\psi=90^{\circ}$, such that

$(\phi, \psi)=(0,90)$

$\varepsilon_{\phi \psi}=\frac{d_{\phi \psi}-d_{0}}{d_{0}}=\varepsilon_{11}$

where $\varepsilon_{11}$ is the in-plane strain (or surface strain) parallel to the fiber direction.

This approach is used because in X-ray diffraction with soft X-rays, diffraction is possible only in reflection and measurements cannot be made at $\psi=90^{\circ}$. In contrast, because of the deep penetration and diffraction taking place in both reflection and transmission geometry in a neutron diffraction experiment, strain in any orientation can be obtained directly, provided a diffraction peak can be found. 


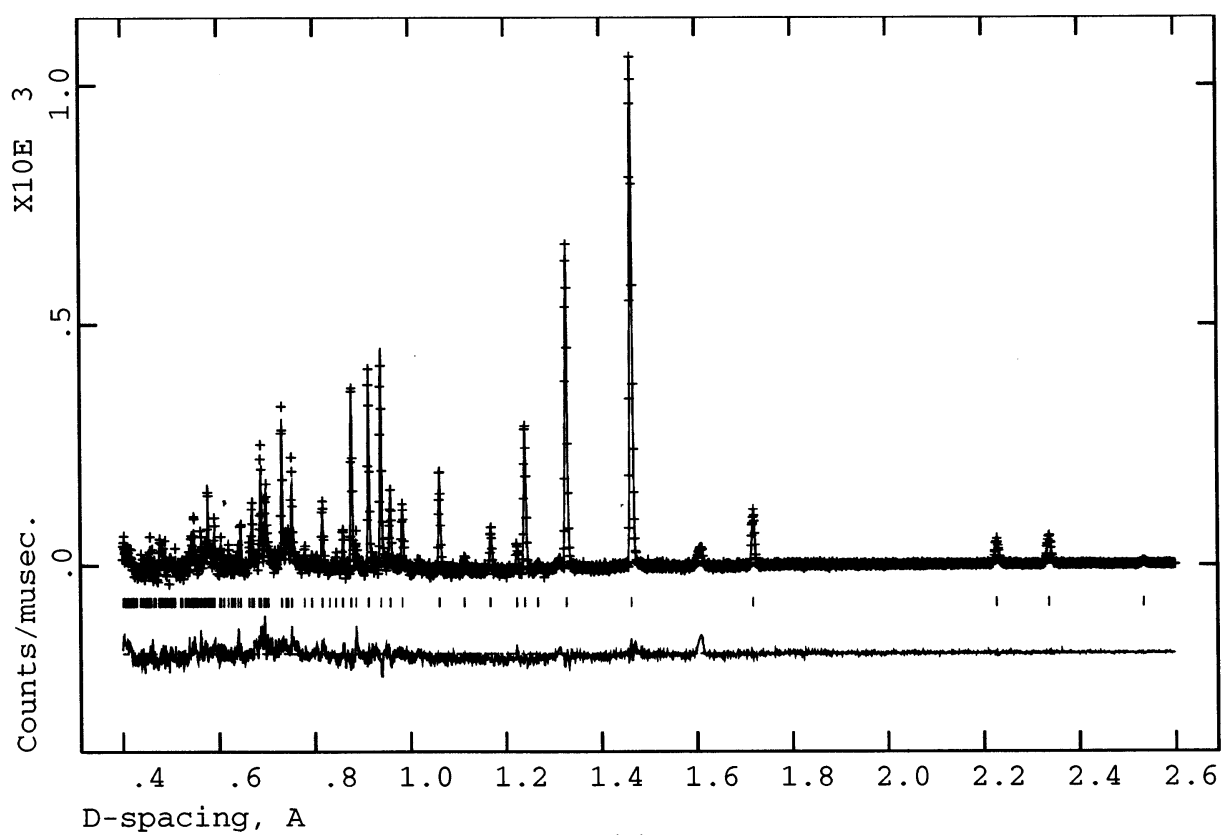

(a)

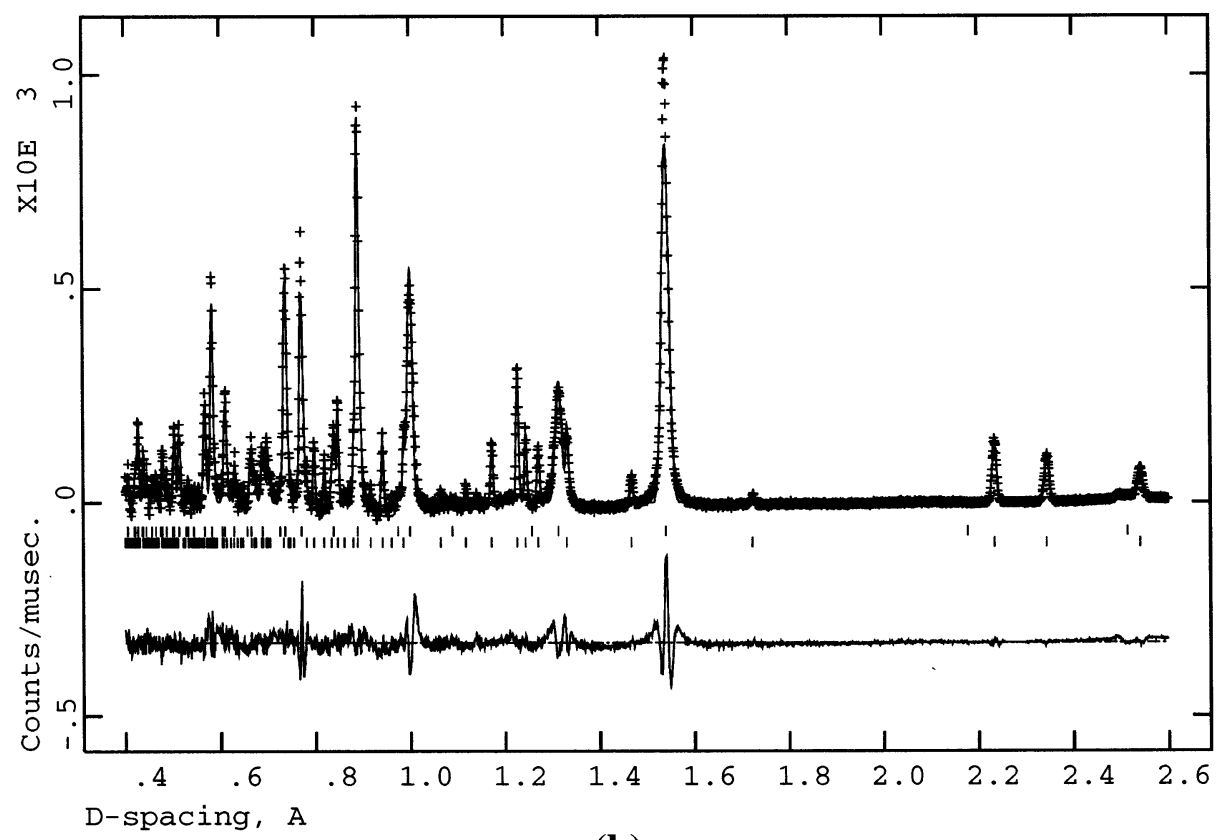

(b)

Fig. 4. Neutron diffraction spectra for the Ti-6Al-4V. (a) Monolithic matrix standard (b) and as-fabricated TMC-with scattering vector parallel to the fiber direction.

\section{Experimental}

\subsection{Sample preparation}

Samples of unidirectional fiber reinforced SCS-6/ Ti-6Al-4V having ten fiber plies were fabricated at Textron Speciality Materials by the foil fiber foil process [19]. A metallographic cross-section, illustrating a typical fiber array, is shown in Fig. 2(a). The spacing is not completely regular and carbon cores can be seen at the center of each fiber. Fig. 2(b) illustrates the typical homogeneous equiaxed $\alpha+\beta$ microstructure of the titanium matrix. The $\beta$ phase shows up as the darker etched lines. The matrix comprised $\sim 90 \% \alpha$ (HCP) and $10 \% \beta$ (BCC) as measured using X-ray diffraction. The $\alpha$ phase of the matrix has a hexagonal close packed 

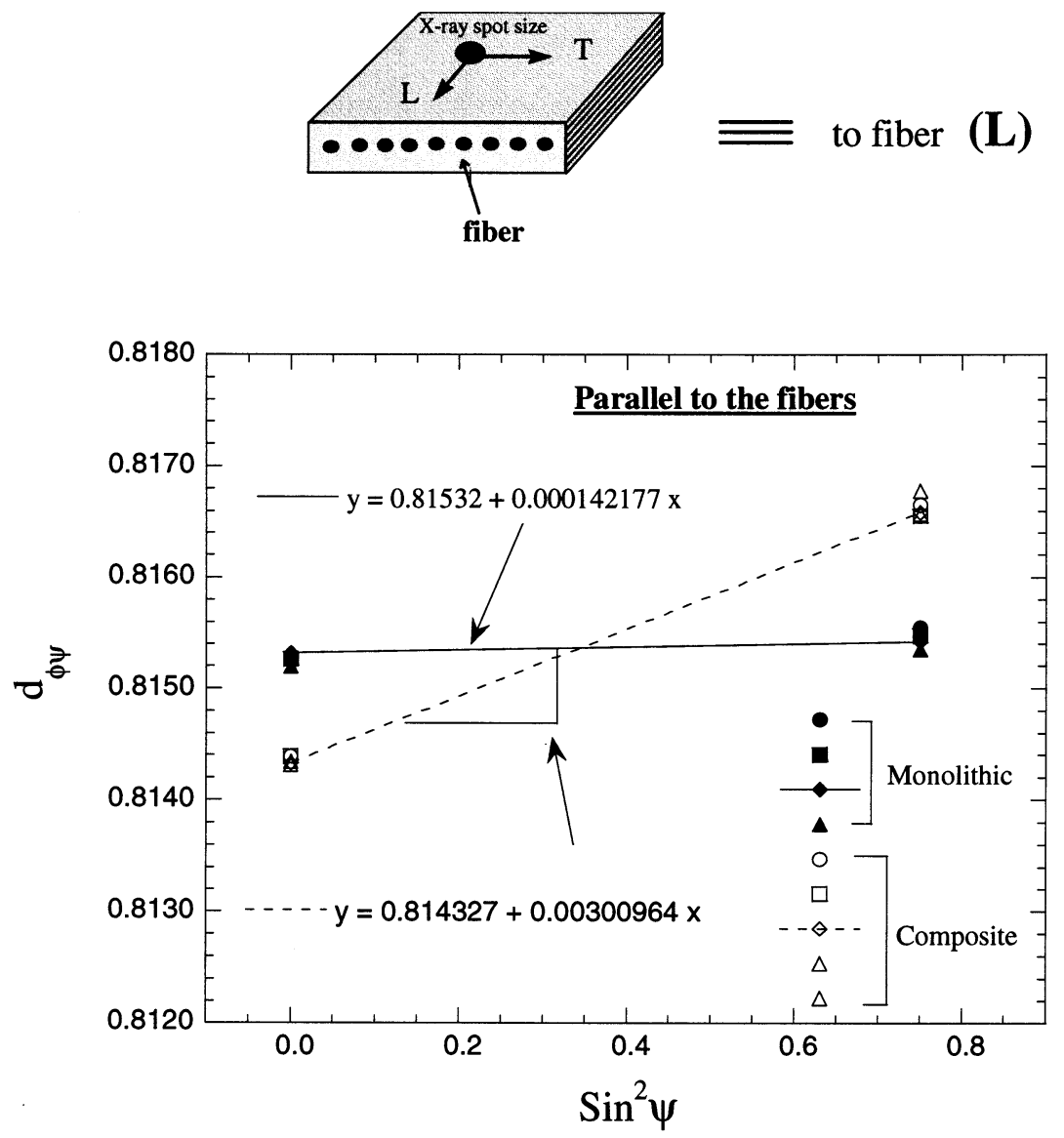

Fig. 5. Plots of $d$-spacing vs. $\sin ^{2} \psi$ for Ti-6Al-4V monolithic alloy and TMC (a) parallel (b) transverse to the fiber direction.

(HCP) crystal structure (space group P $63 / \mathrm{m} \mathrm{m} \mathrm{c}$, lattice parameter $a \approx 2.923 \AA, c \approx 4.667 \AA$ ). The fibers were polycrystalline face centered cubic (FCC) crystal

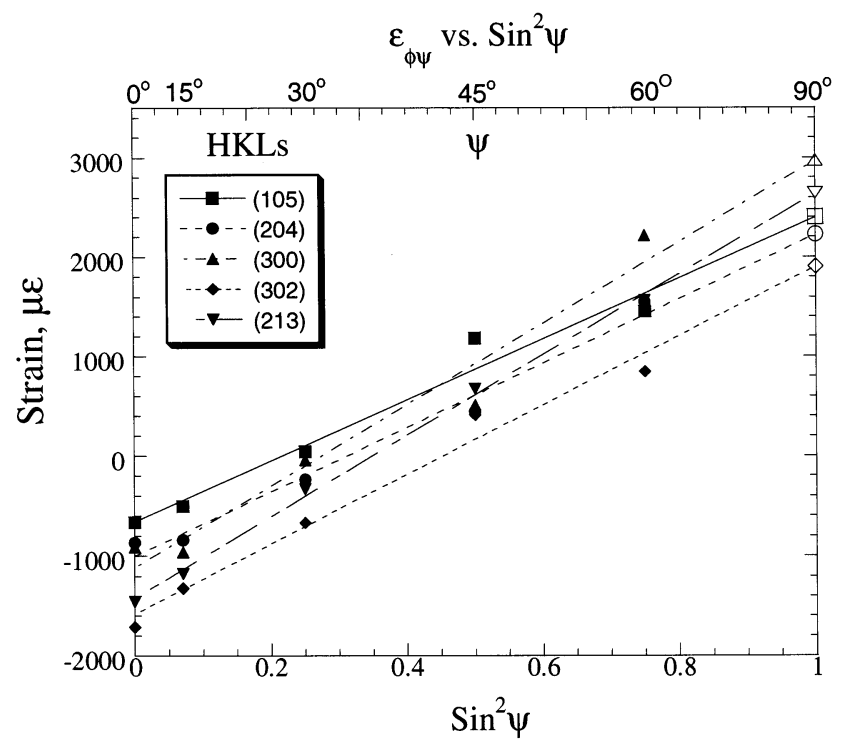

Fig. 6. Plots of $\varepsilon_{\phi \psi}$ vs. $\sin ^{2} \psi$ for hkls 105, 204, 213, 302 and 300, parallel to the fiber direction. Values extrapolated to $\psi=90^{\circ}$ are as open symbols. structure (space group $\mathrm{F}-43 \mathrm{~m}$, lattice parameter $a \approx 4.360 \AA$ ) [20]. Fiber coating layers and the reaction zone at the fiber/matrix interface can also be observed (Fig. 2(b)). The fiber volume fraction $\left(v_{\mathrm{f}}\right)$ was $\approx 37 \%$.

Specimens measuring $100 \mathrm{~mm}$ long, $15 \mathrm{~mm}$ wide and $2 \mathrm{~mm}$ thick, with the fibers oriented in the long direction, were cut from the panels. The specimens were electrochemically polished by a mixture of $6 \%$ perchloric acid, $10 \%$ butyl cellusolve and $84 \%$ methanol at $-50^{\circ}$ at a potential of $50 \mathrm{~V}$. The electropolishing usually resulted in removal of $\sim 10-15$ microns of the matrix material from the surface of each side of the coupon.

\section{2. $X$-ray diffraction}

X-ray measurements used the $\sin ^{2} \psi$ method with $\mathrm{CuK}_{\alpha}$ radiation in the $\alpha(\mathrm{HCP})$ phase of the matrices. The hkls 105, 204, 300, 213, and 302 which scatter at $\approx 123^{\circ}, 128^{\circ}, 132^{\circ}, 142^{\circ}$ and $152^{\circ}$ of $2 \theta$, respectively, were examined (Fig. 3). Only hkls in the high $2 \theta$ $\left(>120^{\circ}\right)$ were used for the analysis because of the greater sensitivity implicit at large angles [17,21]. Measurements longitudinal and transverse to the fibers were made. 


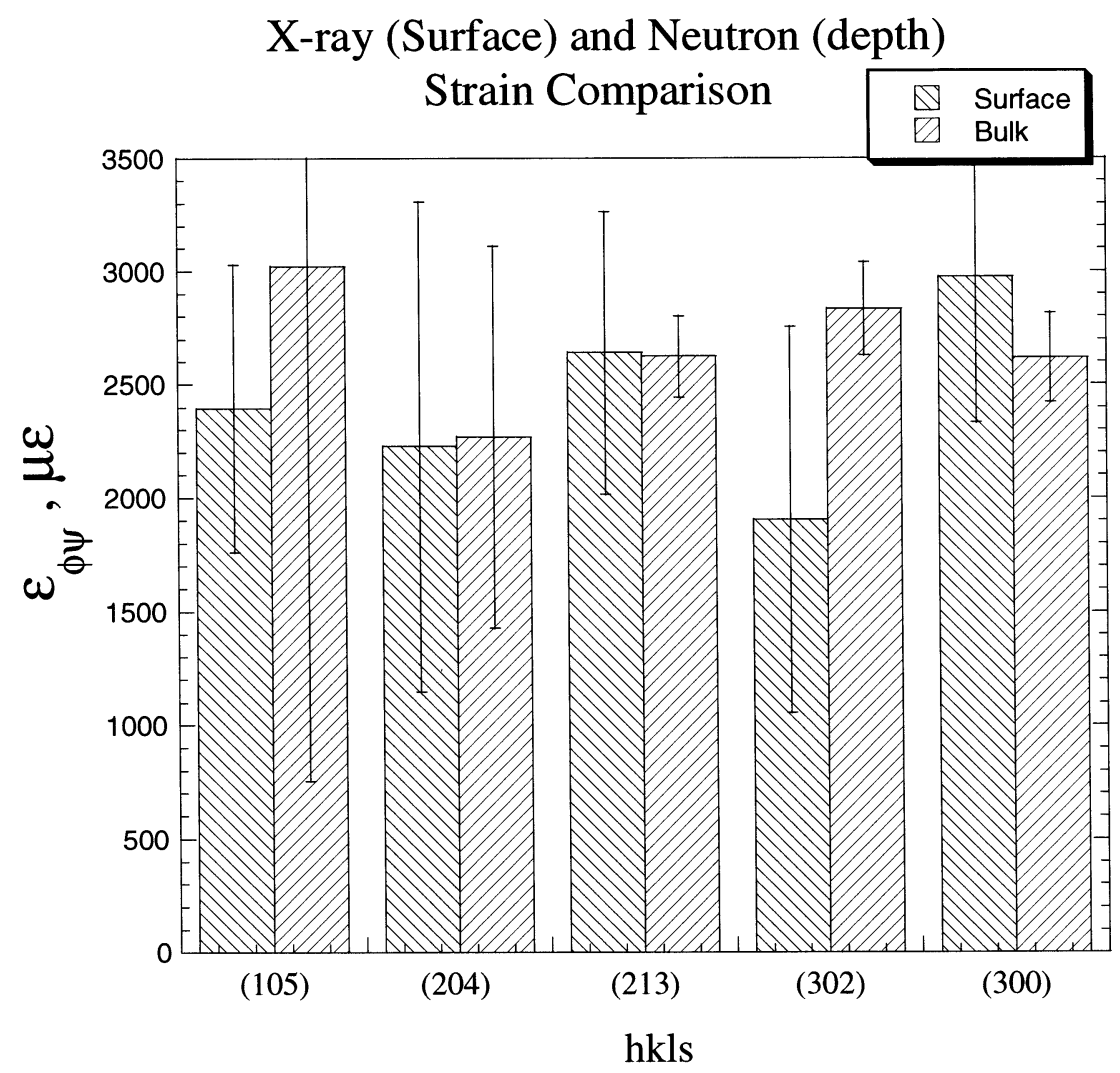

Fig. 7. Average surface X-ray and bulk average neutron elastic strains for hkls 105, 204, 213, 302 and 300.

\subsection{Neutron diffraction}

Neutron strains measurements were made using the neutron powder diffractometer (NPD) at the Manuel Lujan Neutron Scattering Center, as described by Rangaswamy et al. [16]. Average bulk strains $\left(\varepsilon_{\mathrm{hkl}}\right)$ in the composite matrix parallel to the fibers were determined by comparison with stress-free samples of monolithic alloy according to:

$\varepsilon_{\mathrm{hkl}}=\frac{d_{\mathrm{hkl}}-\left(d_{0}\right)_{\mathrm{hk} 1}}{\left(d_{0}\right)_{\mathrm{hkl}}}$

where $d_{\mathrm{hkl}}$ and $\left(d_{0}\right)_{\mathrm{hkl}}$ are the interplanar spacing obtained by fitting individual Bragg reflections in the composite and unstressed standard, respectively, and $\mathrm{hkl}$ are the Miller indices of the diffracting planes in the $\alpha$ (HCP) phase of the matrices. Despite the availability

Table 1

Comparison of X-ray and neutron matrix strains

\begin{tabular}{lllll}
\hline Hkl & X-ray & Error & Neutron & Error \\
\hline$(105)$ & 2396 & \pm 318 & 3022 & \pm 1134 \\
$(204)$ & 2227 & \pm 539 & 2269 & \pm 421 \\
$(213)$ & 2640 & \pm 312 & 2623 & \pm 85 \\
$(312)$ & 1904 & \pm 424 & 2833 & \pm 103 \\
$(300)$ & 2974 & \pm 321 & 2617 & \pm 99 \\
\hline
\end{tabular}

of other hkl reflections, only the five matrix hkl reflections $(105,204,300,213$ and 302) identical to those considered for XRD are reported. Mild texture variations were observed between the titanium in the composites and in the monolithics as seen in Fig. 4(a) and (b). In the fibers the 220 and 440 reflection was used for strain determination. Plane-specific-elastic-strains (PSES) for both composites were calculated using Eq. (6).

\subsection{Results}

\subsection{1. $X$-ray diffraction and neutron diffraction on titanium matrix}

XRD $d_{\mathrm{hkl}}$ versus $\sin ^{2} \psi$ plots were recorded for both the monolithic and the composite matrices. If the monolithic matrix is stress free, then the slopes of the $d_{\mathrm{hk} 1}$ versus $\sin ^{2} \psi$ plot should be close to zero, this is verified as shown in Fig. 5. Our primary intention was comparison of neutron and X-ray longitudinal strains

Table 2

Neutron fiber strains

\begin{tabular}{lll}
\hline Hkl & Neutron & Error \\
\hline$(220)$ & -1273 & \pm 19 \\
$(440)$ & -1220 & \pm 56 \\
\hline
\end{tabular}




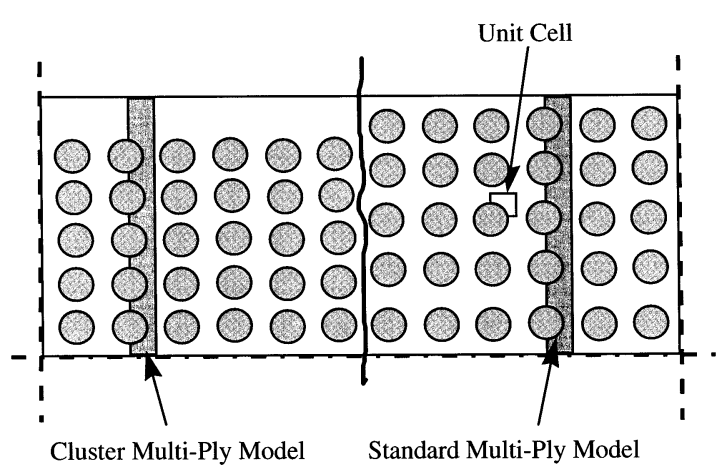

A. Rectangular arrangement of fibers

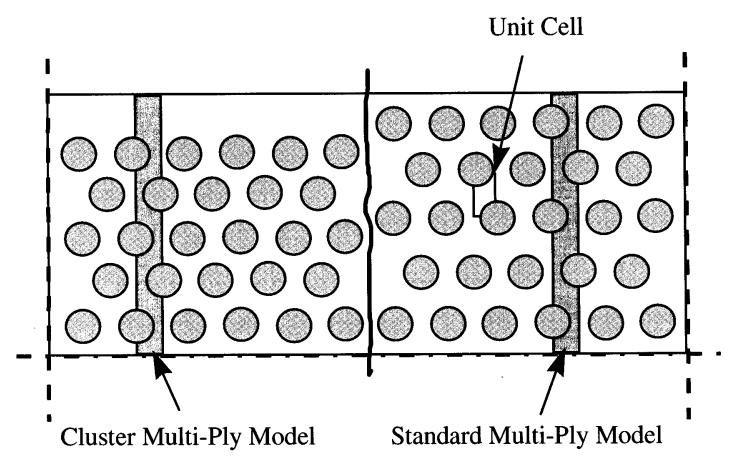

B. Hexagonal arrangement of fibers

Fig. 8. A schematic of the unit cell and multi-ply standard and cluster FEM models for (a) rectangular (b) hexagonal arrangement of fibers.

but $d_{\mathrm{hk} 1}$ versus $\sin ^{2} \psi$ plots were also obtained for the transverse direction. From these, using the approach of Hauk et al. [22], ' $d_{0}$ ' can be determined from both neat and composite matrices. The values differed by $<0.01 \%$.

Fig. 6 shows the $\varepsilon_{\mathrm{hkl}}$ versus $\sin ^{2} \psi$ plots for hkls 105, 204, 300, 213 and 312. The slopes correspond to the difference between in-plane and out-of-plane strains $\left(\varepsilon_{11}-\varepsilon_{33}\right) ; \varepsilon_{11}$ (in-plane strains) is obtained by extrapolation to $\psi=90^{\circ}$. The in-plane strains are plotted in Fig. 7. The hkl strains range from $1904 \pm$
424 to $2974 \pm 321 \mu \varepsilon$, with the 302 plane showing the lowest value and the 300 showing the highest value. The error bars were determined from the standard deviation of measurements at five different locations on the surface and five measurements at the same location.

In neutron diffraction measurements, lattice strains are explicitly determined by measuring $d$ spacings for the composite and the stress-free samples. Since the neutrons irradiate the entire cross-section of the specimen, these are bulk average measurements. The range of neutron determined hkl strains are shown in Fig. 7. Similar to the XRD strains, there is disparity among the hkl strains ranging from $2269 \pm 421$ to $3022 \pm 1134 \mu \varepsilon$, with the (204) plane showing the lowest and the (105) showing the highest values. The error bars in the neutron measurement are a combination of the peak fit error and the average of the strain differences obtained in two consecutive measurements. The large error bar on the 105 plane is due to low peak intensity, resulting in a poor peak fit. Strain values obtained by XRD and neutron diffraction appear to be the same within experimental error as shown in Fig. 7 and Table 1.

\subsubsection{Neutron diffraction on silicon carbide fibers}

The deep penetration of neutrons into the composite sample provides simultaneous measurements of bulk average strains for both the matrix and the fibers. In Table 2 the strains measured in the fibers for hkls 220 and 440 are shown. Since the strains are measured from the same family of planes it is not surprising that they are very similar and within \pm $4 \%$. Since the focus of the present research is on comparing strains for the same hkls measured by Xray and neutrons we have used the fiber strains only as a verification of the strain measurements between the titanium matrix and silicon carbide fibers by checking for stress balance $( \pm 37 \mathrm{MPa})$ in the direction parallel to the fiber orientation.

Table 3

Fiber mechanical properties used in the model

\begin{tabular}{rlll}
\hline Temperature $\left({ }^{\circ} \mathrm{C}\right)$ & Young's Modulus $(\mathrm{GPa})$ & Poisson ratio & Coefficient of thermal expansion $\left(10^{-6} /{ }^{\circ} \mathrm{C}\right)$ \\
\hline 21 & 393 & 0.25 & $3.2025 \mathrm{e}-06$ \\
93 & 390 & 0.25 & $3.3403 \mathrm{e}-06$ \\
204 & 386 & 0.25 & $3.5437 \mathrm{e}-06$ \\
316 & 382 & 0.25 & $3.7369 \mathrm{e}-06$ \\
427 & 378 & 0.25 & $3.9197 \mathrm{e}-06$ \\
538 & 374 & 0.25 & $4.0922 \mathrm{e}-06$ \\
649 & 370 & 0.25 & $4.2543 \mathrm{e}-06$ \\
760 & 365 & 0.25 & $4.4061 \mathrm{e}-06$ \\
871 & 361 & 0.25 & $4.5477 \mathrm{e}-06$ \\
1093 & 354 & 0.25 & $4.7997 \mathrm{e}-06$ \\
\hline
\end{tabular}


Table 4

Ti-6Al-4V mechanical properties used in the model

\begin{tabular}{rlllll}
\hline Temperature $\left({ }^{\circ} \mathrm{C}\right)$ & $\begin{array}{l}\text { Young's Modulus } \\
(\mathrm{GPa})\end{array}$ & Poisson ratio & $\begin{array}{l}\text { Yield stress } \\
(\mathrm{MPa})\end{array}$ & $\begin{array}{l}\text { Flow modulus } \\
(\mathrm{GPa})\end{array}$ & $\begin{array}{l}\text { Coefficient of thermal expansion } \\
\left(10^{-6} /{ }^{\circ} \mathrm{C}\right)\end{array}$ \\
\hline 23 & 125 & 0.31 & 1000 & 0.7 & $8.7800 \mathrm{e}-06$ \\
260 & 110 & 0.31 & 630 & 2.2 & $9.8300 \mathrm{e}-06$ \\
316 & 100 & 0.31 & 630 & 2.2 & $1.0140 \mathrm{e}-06$ \\
427 & 100 & 0.31 & 525 & 2.2 & $1.0710 \mathrm{e}-05$ \\
482 & 80 & 0.31 & 500 & 1.9 & $1.0970 \mathrm{e}-05$ \\
538 & 74 & 0.31 & 446 & 1.9 & $1.1680 \mathrm{e}-05$ \\
650 & 55 & 0.31 & 300 & 1.9 & $1.2210 \mathrm{e}-05$ \\
800 & 27 & 0.31 & 45 & 2 & $1.2290 \mathrm{e}-05$ \\
825 & 20 & 0.31 & 25 & 2 & $1.2370 \mathrm{e}-05$ \\
850 & 5 & 0.31 & 5 & 2 & \\
\hline
\end{tabular}

\section{Finite element modeling}

\subsection{Model generation}

Finite element (FEM) models were used to predict the residual strains using a generalized plane strain approach. Variations in TMC geometry were modeled by considering hexagonal and rectangular arrays, both uniformly spaced and clustered (Fig. 8). Multi-ply models were compared to unit cell models to examine possible differences between bulk and surface strains. For the temperatures and stresses considered in the model, the SiC fibers were treated as elastic, whereas time independent elastic-plastic behavior was used to describe the TMC matrix (Tables 3 and 4). Because creep material properties were not available viscoelastic/viscoplastic behavior was not modeled, therefore, an analysis starting from the processing temperature would predict unrealistically large residual stresses and strains. For this reason, the simulation was started from a stress-free temperature of $700^{\circ} \mathrm{C}$ and cooled to $25^{\circ} \mathrm{C}$ following the work of Kroupa et al. [23]. They showed that by using the Bodner-Partom model, a bilinear elastic-plastic FEM analysis could predict similar residual stresses in a TMC as a viscoelastic/viscoplastic analysis if the stress-free temperature was selected as $\approx 0.7-0.8$ times the absolute processing temperature.

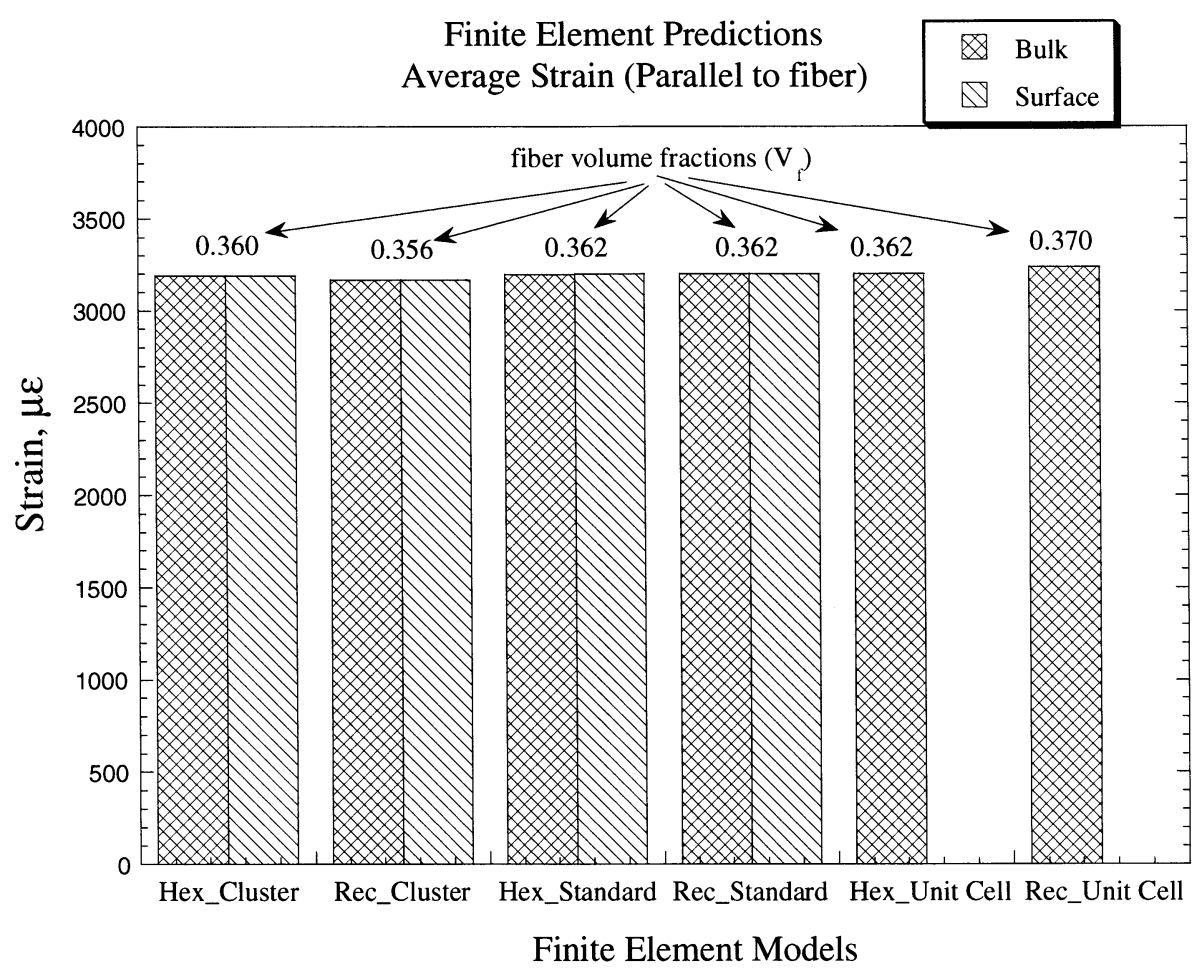

Fig. 9. FEM residual elastic strain predictions for different fiber geometries. 
X-ray, Neutron and Finite Element

Strain Comparison (Parallel to fiber)

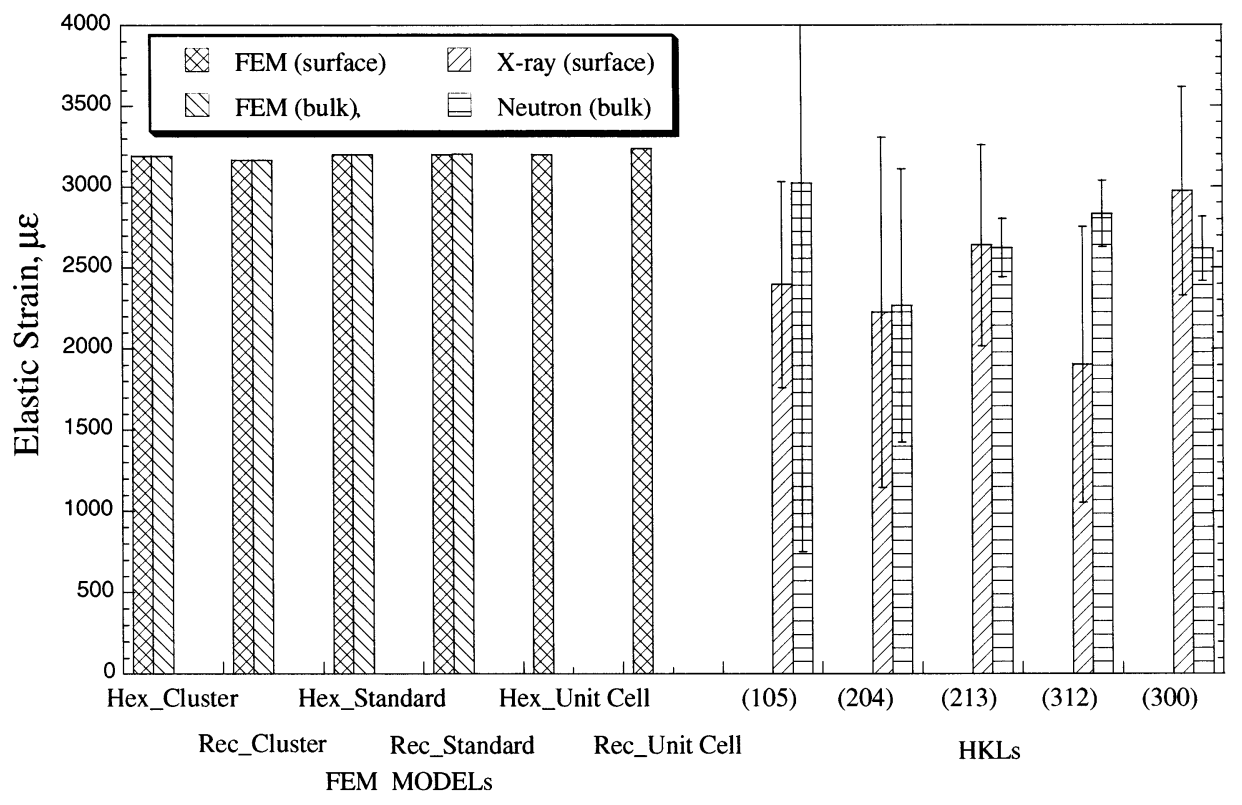

Fig. 10. Comparison of residual elastic strains between measurements and predictions.

\subsection{Modeling results}

The FEM results were post-processed to extract longitudinal matrix strains comparable to the diffraction measurements. Residual strains were volumetrically averaged over the appropriate elements using the elastic strains and deformed shape volume of the elements. Since the maximum penetration depth of X-rays is $\sim 12$ microns at $\psi=0^{\circ}$ and decreases with increasing $\psi$ to 4 microns at $60^{\circ}$, average surface strains were obtained for elements to a depth of 4 microns. FEM strains change negligibly between 4 and 12 microns, so the exact choice of this depth has little influence on the result. The bulk strains for comparison with neutron results were determined using all of the matrix elements in each model. The FEM residual strains for the longitudinal direction in the matrix are shown in Fig. 9.

Surprisingly, all the models showed similar strains of about $+3200 \mu \varepsilon$, and no difference is observed between the surface and bulk. In the figure the exact volume fractions of the fiber reinforcement is listed. Since there are slight variations in the volume fractions, this causes a strain difference between the predictions for the different models. However, the predicted strains are essentially identical for all cases.

\section{Discussion}

\subsection{Philosophy}

Validation of a predictive model TMCs is often performed through experimental determination of stresses. However, this typically ignored the strain anisotropies that are usually apparent when comprehensive diffraction measurements are made. Since researchers involved in finite element modeling predict stresses, they can also predict elastic strains, which are perhaps a more valid comparison since no inferences are made in converting the widely varying elastic strains to stress.

Continuous fiber reinforced TMCs exemplify the complimentary capabilities of XRD and neutron diffraction in characterizing the surface and bulk. In XRD measurements, the presence of a bi-axial stress distribution (supported by a linear $d$ vs. $\sin ^{2} \psi$ plots) strain measurements parallel to the fiber direction simplifies the strain tensor to principal components only. When $d$ versus $\sin ^{2} \psi$ plots are used to convert strains to stresses, a diffraction plane specific elastic constant is used in Eq. (4) $[17,21]$. However, this may be sensitive to the presence of texture which would effect the diffraction elastic constants. 
In contrast, neutron diffraction determines the average mean phase strains in the matrix and fiber of the TMCs. Since these strains are averaged over the entire cross-section of the TMC, there is less ambiguity associated with surface effects. The other advantage of neutron diffraction is that since measurements are available for both matrix and fiber, a stress balance check can be made since all hkl reflections are collected in a measurement at a pulsed source. Since all hkl reflections are collected in a measurement at a pulsed source it forces one to deal with the implications of hkl dependent elastic strain anisotropies. Previously, researchers have simply averaged the strains (neglecting any outliers) for comparison with the continuum FE calculation $[11,16]$. This approach is fraught with assumptions but is intimately coupled with the texture of the material. Moreover, when strong texture is present, a simple average of the strains is arguably not the correct procedure.

\subsection{Comparison of $X R D$ and neutron diffraction}

In Fig. 7, where XRD and neutron strains are compared, there is good agreement between hkls 204 and 213, and reasonable agreement between hkls 105 and 300. However, there is disparity in hkl 302. Although the errors are large enough to make it less than definitive, the hkl strains for the X-ray and neutron measurements show similar trends. This is not entirely surprising, since similar results for other titanium alloy (Ti-6242) composites $[16,24]$ have previously been observed [11].

\subsection{Comparison of experimental versus predictions}

Fig. 10 compares the XRD, neutron and FEM strains. Clearly, the continuum based FEM models predict a single strain value that cannot capture the $\mathrm{hkl}$ variations and the average measured elastic strains are $\sim 500 \mu \varepsilon$ less than the FEM for the stress free temperature of $700^{\circ} \mathrm{C}$. From the FEM models it appears that XRD and neutron diffraction should give identical strains in the longitudinal direction. At least for the $700^{\circ}$ start case which results in no plastic deformation of the matrix with only elastic behavior, the predicted residual longitudinal strains in all the models will agree due to the constraints of thermal expansion and equilibrium.

To explore the implications of partial matrix plasticity, the analyses were repeated using a processing temperature of $900^{\circ} \mathrm{C}$. Although this overestimated the predicted residual strains, it did result in variations between bulk and surface strains for the different models. The differences were only $2-3 \%$ for a given geometry.

\section{Summary and conclusions}

Matrix surface (XRD) and bulk (neutron) strains were compared for 105, 204, 213, 302 and 300 hkls in a $\mathrm{SiC}(\mathrm{SCS}-6) / \mathrm{Ti}-6 \mathrm{Al}-4 \mathrm{~V}$ TMC.

In-plane surface strains were inferred for hkls by extrapolating to $\psi=90^{\circ}$ and comparison with neat material. Parallel to fiber strains were tensile, showing peak to peak variation ranging from $1904 \pm 424$ to $2974 \pm 321 \mu \varepsilon(35 \%)$. Parallel to the fiber bulk strains were also tensile, showing peak to peak variation ranging from $2269 \pm 421$ to $3022 \pm 1134 \mu \varepsilon$. In contrast, the FEM models predicted similar bulk and surface average strains of $3200 \mu \varepsilon$. The $10 \%$ differences between the averaged bulk and averaged surface strains could not be explained by the spatial variation in the finite element predictions.

\section{Acknowledgements}

This work was supported (in part) under the auspices of the US Department of Energy. The Lujan Scattering Center is a national user facility funded by the US Department of Energy, Office of Basic Energy Sciences-Materials Science, under contract number W7405-ENG-36 with the University of California. We acknowledge Jim Larsen and Jay Jira of WL/MLLN for providing the $\mathrm{SiC} / \mathrm{Ti}-6 \mathrm{Al}-4 \mathrm{~V}$ samples and permission to disseminate this information.

\section{References}

[1] P.K. Wright, Thermal stress effects in intermetallic matrix composites, in: E.V. Barrera, I. Dutta, S.G. Fishman (Eds.), Residual Stresses in Composites: Measurement, Modeling and Effect on Thermo-Mechanical Properties, TMS, 1993.

[2] J.M. Larsen, W.C. Revelos, M.L. Gambone, in: D.B. Miracle, D.L. Anton, J.A. Graves (Eds.), Intermetallic Composites II, vol. 273. MRS Proceedings, Pittsburg, PA, 1992, pp 3-16.

[3] H. Gigernzer, P.K. Wright, Plasma sprayed SCS-6/titanium aluminide composite test panels, in: P.R. Smith, S.J. Balsone, T. Nicolas (Eds.), Titanium Aluminide Composites, WL-TR-914020,1989 , pp. $251-264$.

[4] W.S. Johnson, S.J. Lubowinski, A.L. Highsmith, in: J.M. Kennedy, H.H. Moeller, W.S. Johnson (Eds.), Thermal and Mechanical Behavior of Ceramic and Metal Matrix Composites, ASTM STP 1080, Philadelphia, 1990, pp. 193-218.

[5] C.A. Bigelow, W.S. Johnson, A.R. Naik, A comparison of various micromechanics models for metal matrix composites, in: J.N. Reddy, J.L. Telpy (Eds.), Mechanics of Composite Materials and Structures, Book no. HOO464-1989, 1989, pp. 21-31.

[6] M.R. James, Residual stresses in metal matrix composites, in: G. Beck, S. Denis, A. Simon Jr. (Eds.), International Conference on Residual Stresses II, Elsevier, Amsterdam, 1989, pp. 429-435.

[7] P. Rangaswamy, W.C. Revelos, N. Jayaraman, J. Compos. Technol. Res. 16 (1) (January 1994) 47-53.

[8] P. Rangaswamy, N. Jayaraman, J. Compos. Technol. Res. 17 (1) (January 1995) 43-49. 
[9] B.N. Cox, M.R. James, D.B. Marshall, R.C. Addison Jr., Metall. Trans. A 21A (1990) 2701.

[10] M.R. James, Behavior of residual stresses during fatigue of metal matrix composites, in: H. Fujiwara, T. Abe, K. Tanaka (Eds.), Residual Stresses, 3rd, Elsevier, 1992, pp. 555-560.

[11] M.R. James, M.A. Bourke, J.A. Goldstone, A.C. Lawson, Diffraction measurements of residual stress in titanium matrix composites, in: E.V. Barrera, I. Dutta (Eds.), Residual Stresses in Composites, Measurement, Modeling and Effects on ThermoMechanical Behavior, The Minerals, Metals and Materials Society, 1993, pp. 177-185.

[12] A.D. Krawitz, Neutron stress measurements in composites, in: E.V. Barrera, I. Dutta (Eds.), Residual Stresses in Composites, Measurement, Modeling and Effects on Thermo-Mechanical Behavior, The Minerals, Metals and Materials Society, 1993, pp. $161-176$.

[13] A. Saigal, D.S. Kupperman, S. Majumdar, Mater. Sci. Eng. AI50 (1992) 59-66.

[14] S. Majumdar, J.P. Singh, D. Kupperman, A.D. Krawitz, J. Eng. Mater. Technol. 113 (1991) 51-59.
[15] D.S. Kupperman, S. Majumdar, J.P. Singh, Neutron News 2 (1991) $15-18$.

[16] P. Rangaswamy, M.A.M. Bourke, P.K. Wright, E. Kartzmark, J. Roberts, N. Jayaraman, Mater. Sci. Eng. A224 (1997) 200209.

[17] I.C. Noyan, J.B. Cohen, Residual Stress, Springer, New York, 1987.

[18] B. Clausen, T. Lorentzen, T. Leffers, Acta. Mett. 1998 (in-press).

[19] Textron Speciality Materials, Lowell, MA 01851, USA.

[20] C. Jones, C.J. Kiely, S.S. Wang, J. Mater. Res. 4 (2) (1989) 327-335.

[21] B.D. Cullity, Elements of X-Ray Diffraction, 2nd, AddisonWesley, Reading, MA, 1978.

[22] V.M. Hauk, R.W.M. Oudelhoven, G.H.J. Vaessen, Metall. Trans. 13A (1982) 1239.

[23] J.L. Kroupa, R.W. Neu, Compos. Eng. 4 (1994) 965-977.

[24] P. Rangaswamy, M. Daymond, M.A.M. Bourke, R. Von Dreele, K. Bennett, J.A. Roberts, N. Jayaraman, Mater. Sci. Eng. 1998 (in preparation). 